\title{
GAMMA-RAY COMPUTERIZED TOMOGRAPHY USING 10 MEV LASER- COMPTON PHOTON BEAM
}

\author{
\#Hiroyuki Toyokawa, Tomohisa Mikado, Hiroshi Ogawa, Norihiro Sei, Kawakatsu Yamada, \\ Masato Yasumoto, AIST, Tsukuba, Ibaraki 3058568, Japan \\ Hideaki Ohgaki, IAE, Kyoto University, Uji, Kyoto 6110011, Japan \\ Nobutada Aoki, Noriyasu Kobayashi, TOSHIBA PIC, Yokohama, Kanagawa 2300045, Japan
}

\begin{abstract}
We have developed a prototype high energy gamma-ray CT system for nondestructive inspection of heavy industrial products using laser-Compton photon beam, which was generated with an $800 \mathrm{MeV}$ electron storage ring, TERAS, of AIST-Tsukuba and with an intense laser light source. A description of the prototype system and experimental results of $\mathrm{CT}$ imaging are presented.
\end{abstract}

\section{INTRODUCTION}

$\mathrm{X}$-ray radiography is not only used as medical diagnosis tools, but also for industrial purposes, such as nondestructive inspection of industrial products. X-ray Computed Tomography (CT) is one of the most common and powerful tools for these applications. If the X-ray energy is sufficiently high, as high as that of the gammaray energy (typically, higher than a few $\mathrm{MeV}$ ), the X-ray penetrates concretes and metals. High energy photon radiography is, then, a useful tool for a nondestructive inspection of bulk materials and metal die-casts. It is especially useful for an engineering process, so called the reverse engineering, which is one of the methods to improve the efficiency for a development of the industrial products, reducing the time to make various computeraided design models.

Photons with the energies about a few $\mathrm{MeV}$ are useful for the inspection of materials with high atomic numbers (hereafter, high- $Z$ materials), such as iron, copper and lead, because the total attenuation coefficient [1] is minimum around these energies. The attenuation coefficients for lighter materials, or low-Z materials, such as water, tissues and concrete, decrease with increasing photon energy, and are almost constant above $10 \mathrm{MeV}$. Radiography using photons over a few $\mathrm{MeV}$ is, then, almost free from the spectrum hardening effect and the metal artifact, and is useful for the inspection of various industrial products.

Laser Compton scattering (LCS) is one of the techniques to produce high energy intense photon beam [2]. Because the angular spread of the LCS photons is so small, on the order of a few mrad, that high resolution imaging of high- $Z$ materials can be possible. One can obtain photons with good directionality and with narrow spectrum width, at the same time, by putting a collimator along the photon beam line, because the energy of the scattered-off photons via Compton scattering depends on the scattered angle, $\theta_{2}$, as follows:

\footnotetext{
\#h.toyokawa@aist.go.jp; visiting researcher from AIST. Current contact, TJNAF, Newport News, VA 23606, USA.
}

$$
\begin{aligned}
& E_{\gamma}=\frac{E_{L}\left(1-\beta \operatorname{Cos}\left[\theta_{1}\right]\right)}{1-\beta \operatorname{Cos}\left[\theta_{2}\right]+\frac{E_{L}\left(1-\beta \operatorname{Cos}\left[\theta_{2}-\theta_{1}\right]\right)}{E_{e}}} \\
& \text { where } \quad \beta=\sqrt{1-\gamma^{2}}, \quad \gamma=\frac{E_{e}}{0.511}
\end{aligned}
$$

where $E_{\gamma}, E_{L}$ and $E_{e}$ stand for the energies of a LCS photon, a laser quantum and an electron, respectively, in $\mathrm{MeV} . \theta_{1}$ and $\theta_{2}$ are the angles of the laser quantum before and after the Compton scattering, measured with respect to the motion of direction of the electron, where $\theta_{1}$ becomes $\pi$ in the head-on configuration.

Figure 1 shows the energy of a scattered photon as a function of the scattering angle, $\theta_{2}$, assuming the head-on collision $\left(\theta_{1}=\pi\right)$ of a $760 \mathrm{MeV}$ electron and a $1064 \mathrm{~nm}$ laser quantum, calculated from the Klein-Nishina formula. It is understood that energy spread of a few \% can be obtained by confining the angular divergence in a few part of $10 \mathrm{mrad}$.

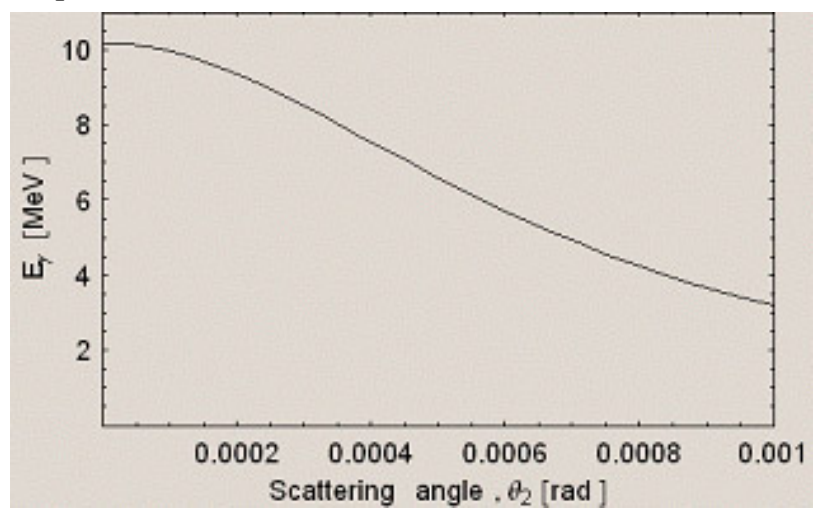

Figure 1: Energy of the $1064 \mathrm{~nm}$ laser quantum scattered via the Compton scattering with the $760 \mathrm{MeV}$ electrons.

\section{EXPERIMENT}

\section{Description of the experimental setup}

We have been developing the LCS photon radiography and CT systems [3,4] at the LCS photon facility of National Institute of Advanced Industrial Science and Technology (AIST) at Tsukuba [5, 6], and installed a prototype CT system, recently. The photon facility serves 1 - $40 \mathrm{MeV}$ quasi-monochromatic and energy-tunable photon beams of up to $10^{6}$ photons $\mathrm{cm}^{-2} \mathrm{~s}^{-1}$ using the 300 $800 \mathrm{MeV}$ electron storage ring, TERAS [7]. Recent experiment showed that the spatial resolution of the high 
energy radiography system using $10 \mathrm{MeV}$ LCS photon beam was better than $1 \mathrm{~mm}[4]$.

The CT experiments were done at the LCS-3 beam line of AIST-LCS photon facility. We used $10 \mathrm{MeV}$ LCS photon beam in the experiment using the commerciallyavailable $\mathrm{Nd}: \mathrm{YVO}_{4}$ laser (SPECTRA-PHYSICS, Millennia IR, TEM00 CW 10W).

Figure 2 shows a schematic view of the LCS-3 beam line of TERAS. The photon beam is generated via the Compton scattering at the center of one of the straight sections of TERAS. The photons go along with the electrons that are swept away at one of the bending magnets of TERAS downstream of the collision point, and go straight through the laser mirror and penetrate it losing negligible numbers of photons. The photon beam is transported about $12 \mathrm{~m}$ in the air to the experimental room. There is a lead collimator of inner diameter of $2.8 \mathrm{~mm}$ and thickness of $100 \mathrm{~mm}$ along the photon beam line. The role of this collimator is to roughly shape the energy spectrum and the beam profile of the LCS photons, and also to suppress the low-energy background photons, which are mainly produced from the bremsstrahlung of the relativistic electrons colliding with the residual gas molecules in the vacuum chamber. The beam divergence and the spot size at the sample, in this configuration, are approximately $0.2 \mathrm{mrad}$ in half angle and $4.5 \mathrm{~mm}$ in diameter, respectively.

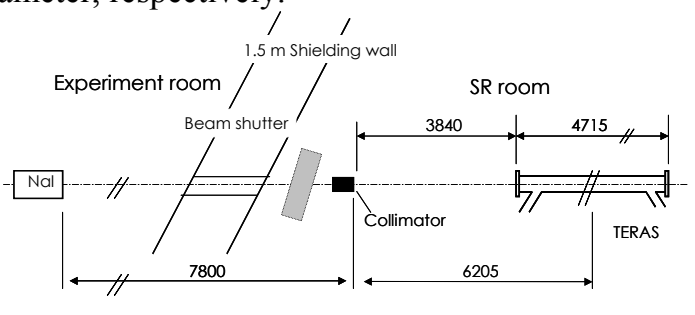

Unit in $\mathrm{mm}$

Figure 2: Schematic drawing of the LCS-3 beam line of AIST-LCS facility.

Figure 3 shows a schematic drawing of the hardware components for the prototype CT system installed in the LCS-3 beam line. Because the diameter and the divergence of the photon beam were very small, we designed the CT system as the $1^{\text {st }}$ generation style. The distance of the sample object from the photon source (laser-electron collision point) is about $12 \mathrm{~m}$. The sample object was put on a PC-controlled CT stage, which had three axes of motion; rotation, horizontal and vertical with respect to the photon beam. A large $\mathrm{NaI}(\mathrm{Tl})$ scintillation detector (8in $x$ 12in) measures the energy and the intensity of the photons. It almost fully absorbs the photons with the energies up to $40 \mathrm{MeV}$. The $2^{\text {nd }}$ collimator, which was placed behind the sample object not only defined the spectrum width and spatial resolution, but also reduced the background photons that were scattered-off from the sample object with small angles via the Compton scattering. We used a collimator of $2 \mathrm{~mm}$ inner diameter. The resulting beam diameter and the divergence are considered to be $1.8 \mathrm{~mm}$ and $80 \mu \mathrm{rad}$, respectively, at the sample position.

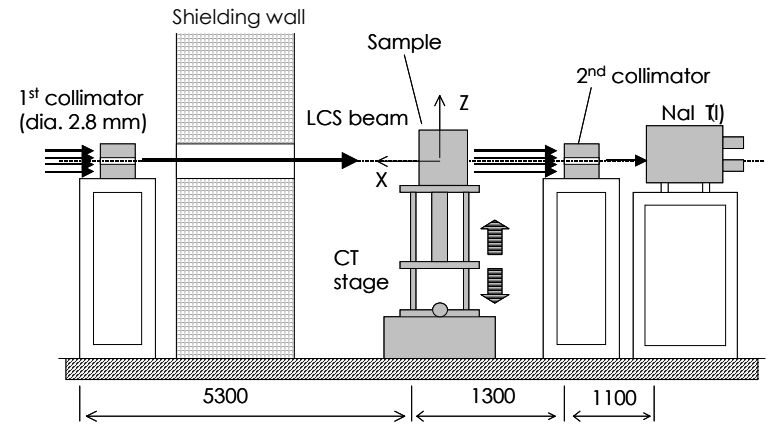

Figure 3: Schematic drawing of the prototype CT system.

\section{Sample object}

We chose the RF tetrode tube "TH571A" (THOMSON TUBES ELECTRONIQUES) as a sample object for the CT experiment, because it consisted of high- $Z$ and low- $Z$ materials and its inside structures were invisible from outside. The anode was put inside the iron vessel, and the cathode and grid structures were inside the ceramics insulator. This tube is usually used as a main amplifier for the RF power source of TERAS.

Figure 4 shows the radiograph of the sample object around the electrodes [4]. The metal grids and cathode are clearly seen through the ceramic insulator. Because we were interested in the structure of the electrode, we decided to take the CT images around these regions. The solid lines indicate the slice positions $(1 \sim 8)$, which were numbered sequentially from bottom to top. The slice thickness and the scanned area were $1 \mathrm{~mm}$ and $100 \times 100$ $\mathrm{mm}^{2}$, respectively.

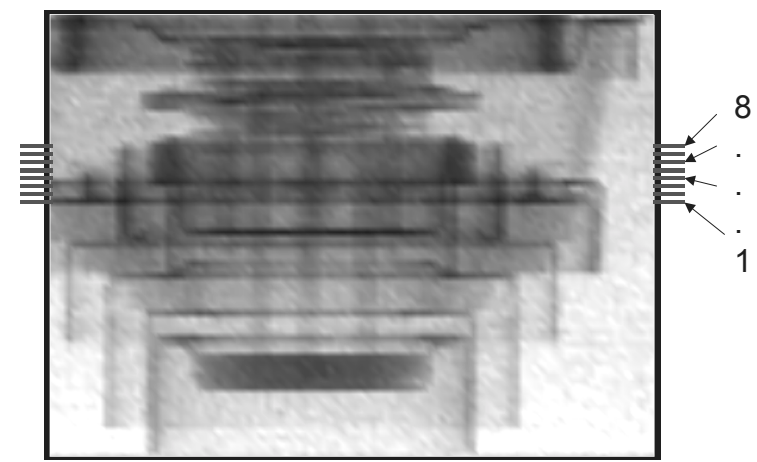

Figure 4: Radiograph around the electrode of the TH571A tetrode tube.

\section{Measurement results}

It took about 1 hour to scan each of the slices. The measurements were done as follows:

1. Rotating the sample object and counting the number of photons within each of the preset angle (3.6 degree, for example) until the object turns 360 degree (which results in 100 angular projection points, for 3.6 degrees increment).

2. Moving horizontally a preset step $(1 \mathrm{~mm}$, for example).

3. Repeating the process 1 and 2, until the total travel distance reaches the preset value $(100 \mathrm{~mm}$, for example). 
4. Completion of a measurement for one slice. Then, moving vertically, and repeating the process $1-3$ for another slice.

Figures 5 show the reconstructed CT images. We can see the cross-sectional views of inside the sample object. The numbers on the top of the images show the vertical positions, or the slice numbers shown in fig. 4. The CT images were reconstructed with the filtered backprojection method, off-line. The count-rate data were Fourier transformed using the Fast Fourier Transformation (FFT) algorithm, and convoluted with the filtering function. We used a step function with the highest cutoff frequency at the Nyquist frequency as the filtering function. The data in the Fourier regime were, then, inversely FFT followed by the coordinate exchange according to the standard tomography method.
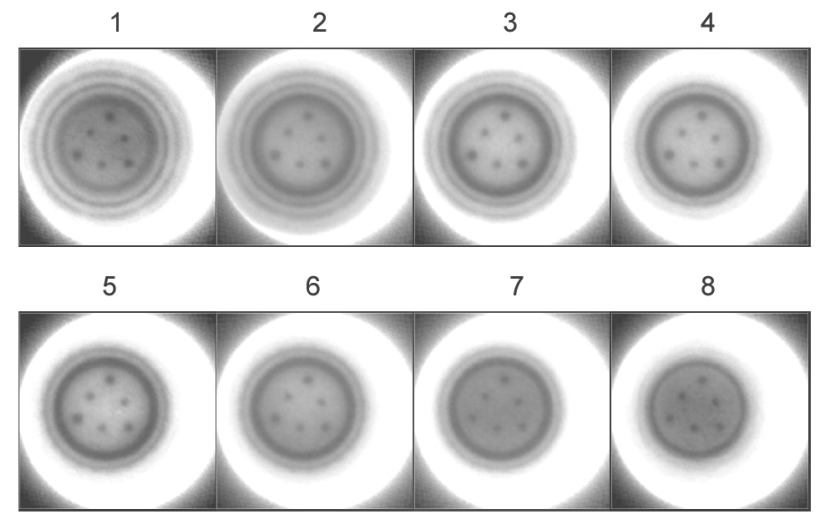

Figure 5: Cross-sectional images of the sample object obtained with the CT using the $10 \mathrm{MeV}$ LCS photon beam.

\section{DISCUSSION}

The present results obtained with the prototype CT system showed that we were able to build the high energy photon CT system for the inspection of bulky industrial products. However, we should improve the present CT system in accordance with the following points to meet with the criteria for a practical use:

1. Reduction of the data acquisition time

2. Optimization of the spatial resolution

The first point is critical, because the current CT system requires 1 hour to obtain an image data for $100 \mathrm{~mm}$ x 100 $\mathrm{mm}$ area. Several ideas to reduce the measurement time have been discussed. Some of them are to increase the photon intensity, and the other ones are to upgrade the system to the second-generation CT system with fanbeam configuration, using multiple detector system. We are interested in how to make the LCS photon beam in a fan-beam configuration, and are preparing for the feasibility study. The second point is also important. Because the space for the CT system installation is limited, some of the optimal geometric conditions to give the best spatial resolution may not be achieved. So, we should evaluate the spatial resolution of the present CT system in the current configuration, first, and, then, try to optimize the system to give the best performance.

\section{SUMMARY}

The high energy photon radiography and CT systems using the $10 \mathrm{MeV}$ laser Compton photon beam of AISTLCS facility has been developed, and the first CT experiment was done. The sample object was the RF tetrode tube made of iron, copper, ceramics, and so on. We were able to obtain the cross-sectional images of the sample object.

\section{ACKNOWLEGEMENTS}

We would like to thank Nikolitsa Merminga of Jefferson Lab for her discussions and encouragement on this work. This study was financially supported by the Budget for Nuclear Research of the Ministry of Education, Culture, Sports, Science and Technology of Japan, based on the screening and counseling by the Atomic Energy Commission of Japan.

\section{REFERENCES}

[1] J. H. Hubbell, "Photon Cross Sections, Attenuation Coefficients, and Energy Absorption Coefficients from $10 \mathrm{keV}$ to $100 \mathrm{GeV}^{\prime \prime}$, NSRDS-NBS 29 (1969).

[2] R. H. Milburn, Phys. Rev. Lett. 10(3), 75-77 (1963).

[3] H. Toyokawa, H. Ohgaki, and T. Shima, IEEE Trans. Nucl. Sci. 49(1), 182-187 (2002).

[4] H. Toyokawa, H. Ohgaki, T. Mikado, K.Yamada, Rev. Sci. Instr. 73(9), 3358-3362 (2002).

[5] H. Ohgaki, H. Toyokawa, K. Kudo, N. Takeda, and T. Yamazaki, Nucl. Instr. and Meth. in Phys. Res. A455, 54-59 (2000).

[6] T. Yamazaki, T. Noguchi, S. Sugiyama, T. Mikado, M. Chiwaki, and T. Tomimasu, IEEE Trans. Nucl. Sci. 32(5), 3406-3409 (1985).

[7] T. Tomimasu, T. Noguchi, S. Sugiyama, T. Yamazaki, T. Mikado, and M. Chiwaki, IEEE Trans. Nucl. Sci.30(4), 3133-3135 (1983) 